\section{Dolor pélvico crónico secundario a síndrome de congestión pélvica. Resultados del tratamiento endovascular de la insuficiencia venosa pelviana y várices genitales}

\author{
OBREN DRAZIC B. ${ }^{1}$, CRISTIAN ZÁRATE B. ${ }^{1}$, FRANCISCO VALDÉS E. ${ }^{1}$,
} RENATO MERTENS M. ${ }^{1}$, MICHEL BERGOEING R. ${ }^{1}$, ALBRECHT KRÄMER S. ${ }^{1}$, LEOPOLDO MARINÉ M. ${ }^{1}$, FRANCISCO VARGAS S. ${ }^{1}$

\section{Embolization of insufficient pelvic veins for pelvic congestion syndrome. Analysis of 17 cases}

Background: Pelvic venous insufficiency may cause pelvic congestion syndrome that is characterized by chronic pelvic pain exacerbated by prolonged standing, sexual activity or menstrual cycle. It may be treated by embolizing the dysfunctional pelvic venous drainage and sometimes resecting vulvar, perineal and thigh varices. Aim: To assess the results of embolization of insufficient pelvic or ovarian veins on pelvic congestion syndrome. Material and Methods: Analysis of 17 female patients aged 32 to 53 years, who underwent subjected to a selective coil embolization of insufficient pelvic and/or ovarian veins through the jugular, basilic or cephalic veins. In the preoperative period, all patients had a lower extremity venous duplex pelvic ultrasound examination and some had an abdominal and pelvic CT angiogram. Results: The technical success of the procedure was $100 \%$ and no complications were registered. During a 32 month follow up, no patient had symptoms of pelvic venous insufficiency or relapse of vulvar or thigh varices. Conclusions: Embolization of insufficient pelvic and ovarian veins is a safe and successful procedure for the treatment of pelvic venous insufficiency or vulvar varices.

(Rev Med Chile 2019; 147: 41-46)

Key words: Dysmenorrhea; Dyspareunia; Pelvic Pain; Varicose Veins; Venous Insufficiency.

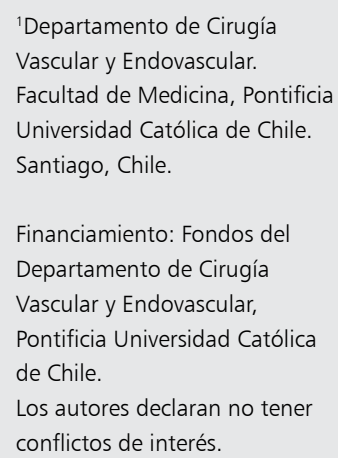

Recibido el 2 de mayo de 2018 aceptado el 10 de enero de 2019

Correspondencia a: José Francisco Vargas Serrano Departamento de Cirugía Vascular y Endovascular. Escuela de Medicina, Pontificia Universidad Católica de Chile. Avenida Diagonal Paraguay 362,

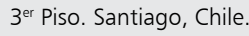
jfvargas@gmail.com
L a insuficiencia venosa pelviana suele manifestarse como un síndrome de congestión pelviana (SCP), que se define como la presencia de dolor pélvico crónico (mayor a seis meses) asociado a insuficiencia demostrada de las venas pélvicas. Este, además, puede tener manifestaciones clínicas que abarcan desde dismenorrea, disuria y dispareunia, hasta la presencia de várices sintomáticas provenientes del territorio pelviano y ubicadas en la región vulvar o cara interna y posterior de muslos, que pueden ser muy sintomáticas. El dolor referido es similar en carácter al producido por las várices de extremidades inferiores (pesadez pelviana exacerbada al estar de pie y de predominio vespertina $)^{1}$.

Esta patología es una causa importante del síndrome de dolor pélvico crónico, el cual es responsable de alrededor de $30 \%$ de las consultas 
ginecológicas ambulatorias en Estados Unidos de Norteamérica, pudiendo afectar hasta a $40 \%$ de las mujeres durante toda su vida². También se describe como causa frecuente de recidiva varicosa posterior a cirugía de insuficiencia venosa de extremidades inferiores ${ }^{3}$.

El tratamiento se compone de dos etapas: 1) Evitar el reflujo venoso mediante una etapa endovascular que consiste en la embolización selectiva de los paquetes venosos pelvianos insuficientes y 2) la posterior resección quirúrgica de las várices vulvares o del muslo.

Nos planteamos evaluar los resultados del tratamiento del SCP mediante embolización selectiva de las venas ováricas, pelvianas o ambas insuficientes y posterior resección de los paquetes varicosos de la región vulvar o muslo. Además, evaluamos su impacto en la calidad de vida de estas pacientes a largo plazo.

\section{Materiales y Métodos}

Se evaluaron retrospectivamente los registros clínicos de las pacientes sometidas al tratamiento de insuficiencia venosa pelviana entre junio de 2007 y abril de 2017 por médicos del Departamento de Cirugía Vascular y Endovascular de la Pontificia Universidad Católica de Chile. Se obtuvo aprobación del comité de ética institucional para este estudio.

Se consideró éxito técnico el identificar y embolizar al menos una vena insuficiente durante la flebografía.

Se efectuó, posteriormente, una encuesta telefónica donde se consultó sobre dispareunia, dolor pélvico y reaparición de várices visibles. En esta se definió como éxito a largo plazo la ausencia de síntomas pélvicos o dispareunia y la ausencia de várices vulvares a los 12 meses posterior a la cirugía.

\section{Resultados}

Fueron tratadas 17 pacientes con SCP. El promedio de edad al momento de consulta fue de 41 Años. Todas ellas habían tenido al menos 1 embarazo (mediana 3, rango 1 a 8). El estudio preoperatorio incluyó ecografía dúplex venosa de extremidades inferiores en todas y angiotac de abdomen y pelvis en fase venosa en seis casos. La única paciente previamente histerectomizada fue estudiada con ecografía transvaginal, que confirmó la presencia de várices pelvianas. Las restantes pacientes fueron intervenidas basándose en su ecografía dúplex de extremidades inferiores que confirmaba origen pelviano de su insuficiencia venosa (Tabla 1 ).

Todas las pacientes presentaban várices visibles en extremidades inferiores al momento de consultar. Dos pacientes $(11,8 \%)$ habían sido previamente operadas por várices en extremidades inferiores y presentaban recidiva. Diez de ellas referían dolor pelviano crónico exacerbado en período menstrual y 5 presentaban dispareunia caracterizada por dolor pelviano y sensación de pesadez en relación con paquetes varicosos en la región vulvar.

Se utilizó la clasificación CEAP para estandarizar el registro de las várices de extremidades inferiores ${ }^{4,5}$. Esta clasificación fue desarrollada en

Tabla 1. Características de los pacientes

\begin{tabular}{|lc|}
\hline $\mathrm{n}$ de participantes & 17 \\
\hline Edad & $41(35-53)$ \\
\hline Embarazos, mediana (rango) & $3(1-8)$ \\
\hline Tabaquismo & \\
$\quad$ Activo & $2(11,8 \%)$ \\
$\quad$ Suspendido & $4(23,5 \%)$ \\
Duración de várices años, media (rango) & $6,6(2-15)$ \\
Várices visibles & \\
$\quad$ Muslos & $16(94,1 \%)$ \\
Vulvares & $6(35,3 \%)$ \\
$\quad$ Inguinales & $3(17,6 \%)$ \\
Glúteas & $2(11,8 \%)$ \\
Sin venas visibles & 0 \\
Dolor pélvico preoperatorio & $10(59 \%)$ \\
\hline Dispareunia preoperatoria & $5(29 \%)$ \\
Clasificación CEAP & \\
C2EpAsPr & $14(82,4 \%)$ \\
C2EpApPr & $3(17,6 \%)$ \\
Estudio preoperatorio & $17(100 \%)$ \\
Ecografía & $6(35,3 \%)$ \\
AngioCT & $1(5,9 \%)$ \\
\hline Ecografía transvaginal & \\
\hline
\end{tabular}


1994 por el Foro Venoso Americano (American Venous Forum) y avalado por la Sociedad para Cirugía Vascular (Society for Vascular Surgery) para estandarizar la publicación de resultados de trabajos en patología venosa. En la Tabla 2 se explica lo que significa cada una de sus siglas. Todas las pacientes tenían várices visibles sin edema ni cambios en la piel (categoría Clínica 2). Todas las pacientes tenían etiología primaria de su insuficiencia venosa (Categoría Etiológica p). De las 17 pacientes, 3 presentaban insuficiencia de venas perforantes (Categoría Anatómica p) y 14 presentaban solo insuficiencia superficial (Categoría Anatómica s). Todas las pacientes presentaban várices por reflujo (categoría fisiopatológica r). El tratamiento consistió en la embolización percutánea selectiva de una o más venas ováricas y venas hipogástricas insuficientes mediante coils utilizando la vena yugular interna (14 pacientes), vena basílica ( 2 pacientes) o cefálica ( 1 paciente) como acceso (Figuras 1 y 2 ) (Tabla 3 ).

En $13(76,5 \%)$ pacientes se realizó safenectomía o resección de colaterales en el mismo tiempo quirúrgico, lo que se relaciona a que en 9 casos $(52,9 \%)$ se requirió anestesia general.

El éxito técnico fue 100\% (Figuras 1 y 2). No hubo complicaciones intraoperatorias, locales ni sistémicas.

Todas las pacientes fueron dadas de alta al día siguiente de la intervención y no se registraron complicaciones postoperatorias. Cinco pacientes acusaron dolor tipo pesadez en la región pelviana por aproximadamente 5 días luego de la embolización, que luego cedió completamente.
Tabla 2. Clasificación CEAP

\begin{tabular}{|c|c|c|}
\hline \multirow{10}{*}{\multicolumn{2}{|c|}{ C Clínica }} & CO : Sin signos visibles ni palpables \\
\hline & & C1 : Telangectasias o venas reticulares \\
\hline & & C2 : Várices \\
\hline & & C3 : Edema \\
\hline & & C4a: Pigmentación o Eczema \\
\hline & & $\begin{array}{l}\text { C4b: Lipodermatoesclerosis o atrofia } \\
\text { blanca }\end{array}$ \\
\hline & & $\begin{array}{l}\text { C5 : Cambios cutáneos con úlcera } \\
\text { cicatrizada }\end{array}$ \\
\hline & & C6 : Cambios cutáneos con úlcera activa \\
\hline & & A : Asintomático \\
\hline & & S : Sintomático \\
\hline \multirow[t]{4}{*}{$E$} & Etiología & Ec : Congénita \\
\hline & & Ep : Primaria \\
\hline & & $\begin{array}{c}\text { Es : Secundaria (postraumática o } \\
\text { postrombótica) }\end{array}$ \\
\hline & & En : Sin etiología venosa identificada \\
\hline \multirow[t]{4}{*}{ A } & Anatomía & As : Venas del sistema superficial \\
\hline & & Ad : Venas del sistema profundo \\
\hline & & Ap : Venas perforantes \\
\hline & & An : Sin localización identificada \\
\hline \multirow[t]{4}{*}{$P$} & Fisiopa- & $\operatorname{Pr} \quad$ : Reflujo \\
\hline & tología & Po : Obstrucción \\
\hline & & Pro : Reflujo y obstrucción \\
\hline & & Pn : Sin fisiopatología venosa identificada \\
\hline
\end{tabular}

Referencia: Eklöf B, Rutherford RB, Bergan JJ, Carpentier PH, Gloviczki P, Kistner RL, Meissner MH, Moneta GL, Myers K, Padberg FT, Perrin M, Ruckley CV, Smith PC, Wakefield TW, American Venous Forum International Ad Hoc Committee for Revision of the CEAP Classification Revision of the CEAP classification for chronic venous disorders: consensus statement ${ }^{5}$.

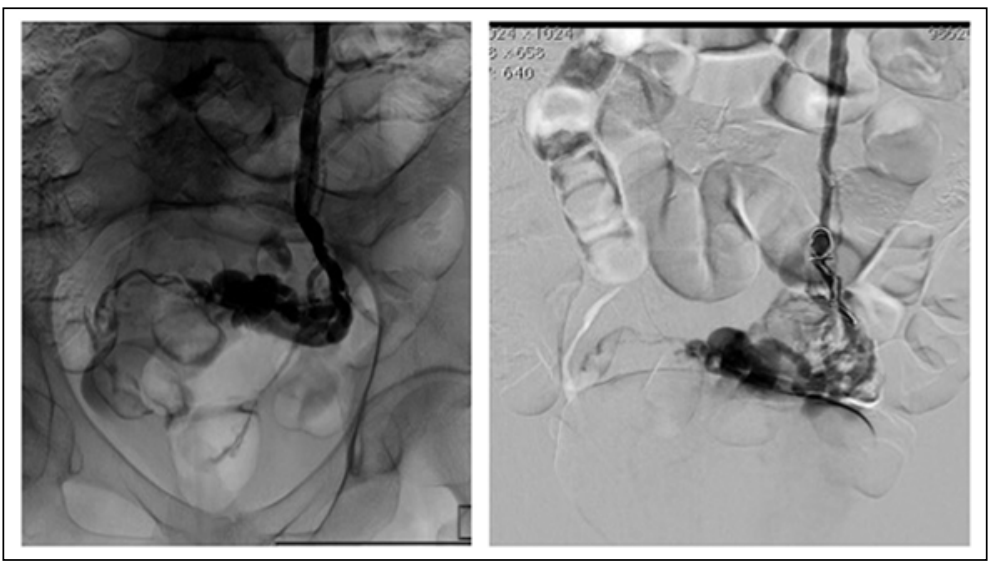

Figura 1. Flebografía selectiva que confirma llene de paquetes varicosos pelvianos dependientes de la vena ovárica izquierda y su posterior embolización. 


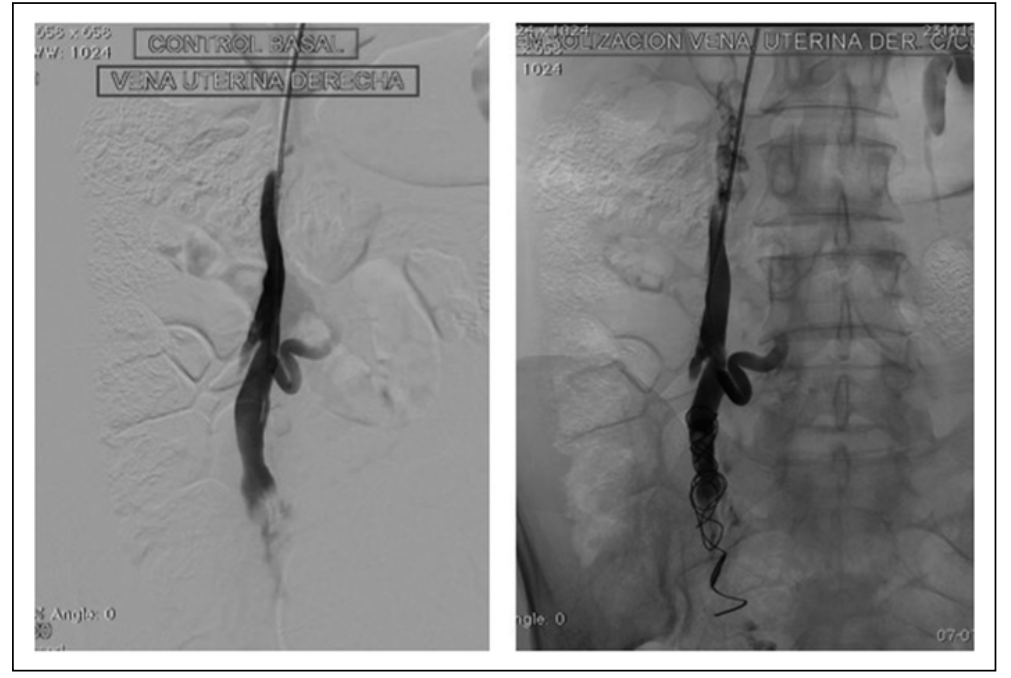

Figura 2. Flebografía de vena iliaca derecha y posterior embolización de las venas insuficientes.
Tabla 3. Variables operatorias

\begin{tabular}{|lc|}
\hline $\begin{array}{l}\text { Anestesia } \\
\text { Local } \\
\text { General }\end{array}$ & $9(52,9 \%)$ \\
Acceso & $8(47,1 \%)$ \\
VYID & \\
VP & $14(82,4 \%)$ \\
Vena embolizada & $3(17,6 \%)$ \\
Ambas venas ováricas & \\
VOD & $9(52,9 \%)$ \\
VOI & $1(5,9 \%)$ \\
Cantidad de coils, mediana & $7(41,2 \%)$ \\
Días hospitalización, media & $4(1-12)$ \\
Éxito técnico & 1 \\
\hline
\end{tabular}

Se logró el seguimiento alejado de 14 de las 17 pacientes $(82,4 \%)$, (promedio: 32 meses). Para evaluar el resultado a largo plazo se contactó por vía telefónica a las 14 pacientes. Ninguna de ellas había presentado recidiva varicosa vulvar ni habían presentado síntomas sugerentes de insuficiencia venosa pelviana. Cuatro pacientes presentaban recidiva varicosa en extremidades inferiores, todas C1EsApPr según clasificación CEAP, sin reintervención. Una de las pacientes había cursado 3 nuevos embarazos sin contratiempos ni recidiva.

\section{Discusión}

El síndrome de congestión venosa pelviana (SCP) es un cuadro de dolor abdominopelviano vago, difícil de caracterizar, secundario a insuficiencia venosa ovárica y de venas hipogástricas, siendo homólogo al cuadro de varicocele $e^{6}$. En el hombre es fácilmente detectable al examen físico y su tratamiento mediante cirugía con ligadura de los paquetes varicosos es habitualmente sencillo, al contrario del SCP en la mujer, en las que puede ser un diagnóstico más esquivo.

El cuadro fue descrito por primera vez en 1949 por Taylor y, desde entonces, su rol etiológico en cuadros de dolor pelviano crónico se ha ido aclarando ${ }^{7}$. Las venas ovárica izquierda e iliaca interna derecha son las más afectadas (hasta 60\% de las pacientes) y más de la mitad de las pacientes tendrán dos o más ejes venosos afectados ${ }^{8}$.

Las pacientes pueden presentar várices visibles en la región vulvar o perianal y en la parte alta, medial o posterior del muslo ${ }^{9}$. Esto último hace que muchas veces sean tratadas como insuficiencia venosa de extremidades inferiores con ablación de los ejes safenos o safenectomía, lo que no resuelve el cuadro de dolor pelviano ni las várices; las que recidivan en breve plazo. 
Las várices no serán visibles en la gran mayoría de las pacientes. Es por esto que el diagnóstico debe sospecharse en mujeres multíparas (más de 2 embarazos) que consultan por dolor de tipo pesadez pelviana que es más intenso en las tardes y se intensifica luego de mantener relaciones sexuales y previo a la menstruación. En estas situaciones es atribuible al aumento del flujo sanguíneo a la región genital que ocurre en esos períodos ${ }^{10}$.

El diagnóstico no se logra fácilmente con estudios de imágenes tradicionales (ecografía o tomografía computada), ya que estos se hacen con las pacientes en decúbito y no de pie y sin solicitar maniobras de Valsalva ${ }^{11,12}$; maniobra que aumenta la presión venosa. En los angiotac en fase venosa realizados a seis de nuestras pacientes no se logró informar por el radiólogo la presencia de venas insuficientes, pero era posible visualizar grandes cordones varicosos periuterinos o dilatación de una o ambas venas ováricas. El estudio con venografía por resonancia nuclear magnética o flebografía son los más útiles ${ }^{13}$, siendo este último el gold standard para el diagnóstico, además de tener la ventaja de permitir realizar tratamiento mediante la embolización de las venas insuficientes ${ }^{9,14}$. Así lo confirmamos en nuestras pacientes; en todas se detectaron venas ováricas, pelvianas o ambas insuficientes con la flebografía.

Nuestros buenos resultados inmediatos, comprobados con un éxito de $100 \%$ en los procedimientos, asociado a nulas complicaciones y recurrencia, confirman que el tratamiento de la insuficiencia venosa pelviana mediante embolización selectiva de las venas insuficientes (ováricas, hipogástrica o ambas), asociado o no a la resección quirúrgica de paquetes varicosos vulvares cuando estos están presentes, es un tratamiento efectivo y seguro para el manejo de mujeres con dolor pelviano crónico por esta causa.

Inicialmente, nuestro equipo realizaba los dos procedimientos por separado, pero una vez confirmada la seguridad y nula morbilidad, se comenzó a realizar embolización y cirugía en un solo tiempo.

\section{Conclusión}

La insuficiencia venosa pelviana es una entidad diagnóstica que debe detectarse y diferenciarse de la insuficiencia venosa superficial de extremidades inferiores.
La embolización de venas ováricas es un procedimiento mínimamente invasivo seguro, que permite el tratamiento de la insuficiencia venosa pelviana y que, combinado con la resección de las várices vulvares o de ubicaciones atípicas en el muslo, permite lograr excelentes resultados a corto y largo plazo.

\section{Referencias}

1. Ganeshan A, Upponi S, Hon LQ, Uthappa MC, Warakaulle DR, Uberoi R. Chronic pelvic pain due to pelvic congestion syndrome: The role of diagnostic and interventional radiology. Cardiovasc Intervent Radiol 2007; 30 (6): 1105-11.

2. O'Brien MT, Gillespie DL. Diagnosis and treatment of the pelvic congestion syndrome. J Vasc Surg Venous Lymphat Disord 2015; 3 (1): 96-106.

3. Meneses L, Fava M, Díaz P, Andía M, Tejos C, Irarrazaval $\mathrm{P}$, et al. Embolization of incompetent pelvic veins for the treatment of recurrent varicose veins in lower limbs and pelvic congestion syndrome. Cardiovasc Intervent Radiol 2013; 36 (1): 128-32.

4. Porter JM, Moneta GL. Reporting standards in venous disease: an update. International Consensus Committee on Chronic Venous Disease. J Vasc Surg 1995; 21 (4): 635-45. http://www.ncbi.nlm.nih.gov/pubmed/7707568. (Accessed April 18, 2018).

5. Eklöf B, Rutherford RB, Bergan JJ, Carpentier PH, Gloviczki P, Kistner RL, et al. Revision of the CEAP classification for chronic venous disorders: Consensus statement. J Vasc Surg 2004; 40 (6): 1248-52.

6. Liddle AD, Davies AH. Pelvic congestion syndrome: chronic pelvic pain caused by ovarian and internal iliac varices. Phlebology 2007; 22 (3): 100-4.

7. Taylor HC. Vascular congestion and hyperemia; their effect on function and structure in the female reproductive organs; the clinical aspects of the congestion-fibrosis syndrome. Am J Obstet Gynecol 1949; 57 (4): 637-53. http://www.ncbi.nlm.nih.gov/pubmed/18113695.

8. Asciutto G, Asciutto KC, Mumme A, Geier B. Pelvic Venous Incompetence: Reflux Patterns and Treatment Results. Eur J Vasc Endovasc Surg 2009; 38 (3): 381-6.

9. Ignacio E, Dua R, Sarin S, Harper AS, Yim D, Mathur V, et al. Pelvic Congestion Syndrome: Diagnosis and Treatment. Semin Intervent Radiol 2008; 25 (4): 3618.

10. Moore CJ. Pelvic congestion syndrome. Endovasc Interv A Case-Based Approach. 2014; pp. 1247-51.

11. Monedero JL. The role of duplex ultrasound in the wor- 
kup of pelvic congestion syndrome. Phlebolymphology 2015; 22 (1): 44.

12. Harris RD, Holtzman SR, Poppe AM. Clinical outcome in female patients with pelvic pain and normal pelvic US findings. Radiology 2000; 216 (2): 440-3.

13. Meneses QL, Tejos NC, Irarrazabal MP. Utilización de la Resonancia Magnética con contraste de fase para el diagnóstico del Síndrome de Congestión Pelviana. Rev Chil Radiol 2009; 15: 54-8.

14. Daniels JP, Champaneria R, Shah L, Gupta JK, Birch J, Moss JG. Effectiveness of Embolization or Sclerotherapy of Pelvic Veins for Reducing Chronic Pelvic Pain: A Systematic Review. J Vasc Interv Radiol 2016; 27 (10): 1478-86.e8. 